\title{
Writing as a Form of Freedom and Happiness Celebrating the 60th birthday of Gheorghe Păun
}

\author{
G. Ciobanu
}

Gabriel Ciobanu

Romanian Academy, Institute of Computer Science

and A.I.Cuza University of Iasi, Romania

E-mail: gabriel@info.uaic.ro

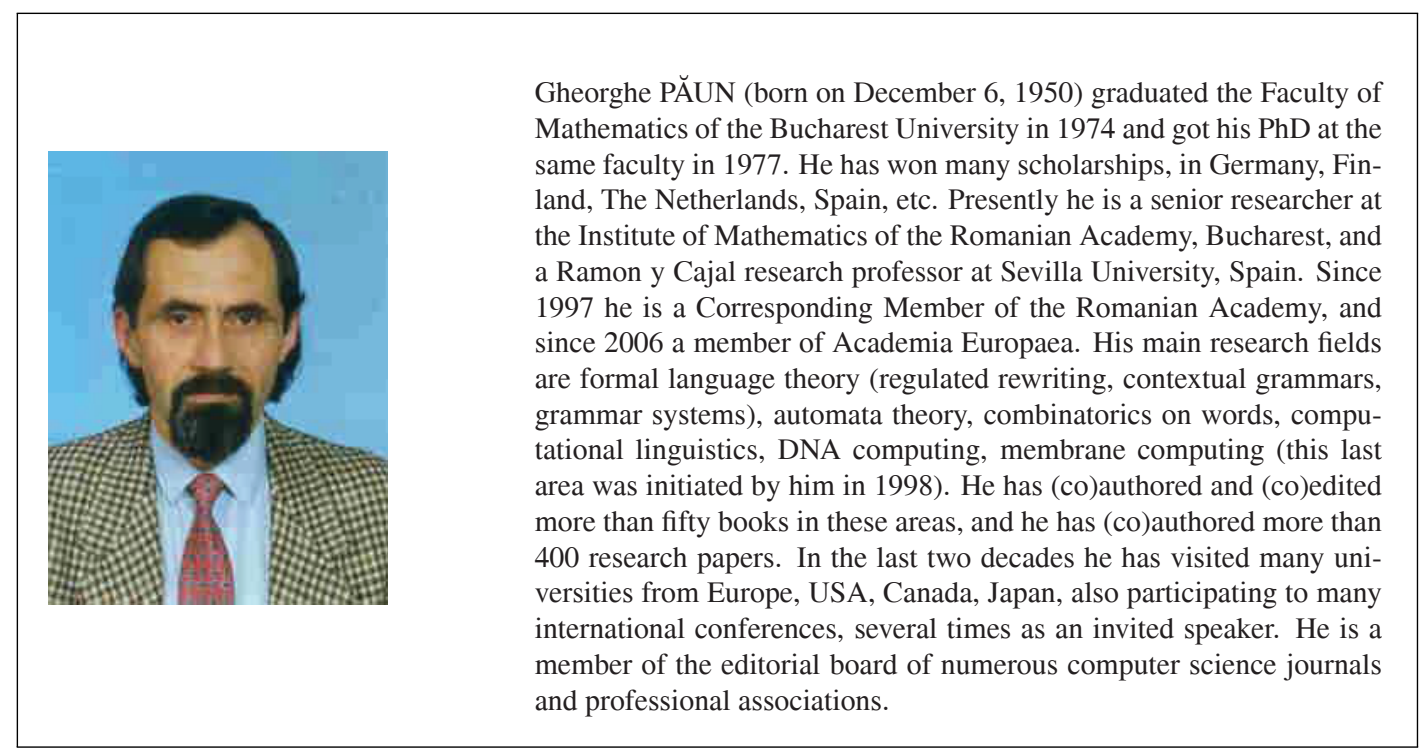

Figure 1: A copy cropped from [1]

Essentially writing is form of thinking on paper, and a way of learning. According to Winston Churchill, writing a book is an adventure. "To begin with, it is a toy and an amusement; then it becomes a mistress, and then it becomes a master, and then a tyrant. The last phase is that just as you are about to be reconciled to your servitude, you kill the monster, and fling him out to the public." On the other hand, writing could be a form of freedom by escaping the madness of a period, and reducing the anxiety. In many situations the authors write to save themselves, to survive as individuals.

Gheorghe Păun is an example of a person affirming his own existence by writing. He is a prolific writer with a huge number of papers: tens of scientific books, hundreds of articles, several novels, poems, and books on games. A list of his scientific publications is posted at http://www.imar.ro/ gpaun/papers.php [2], while his books are listed at http://www.imar. ro/ gpaun/books . php [1] His way of distributing information is not by speaking, but by writing. Gheorghe Păun did not like very much to teach in universities. He preferred a form of "teaching by researching", combining ideas with nice metaphors and distributing his knowledge in articles and books. In this way he wrote several papers having a high impact in the scientific community. His seminal paper "Computing with membranes" published in Journal of Computers and System Sciences in 2000 and his fundamental book on computation theory "Membrane Computing" (Springer, 2003) has over 1,000 citations [6] (and his author was recognized as an "ISI highly cited researcher" [5]). He has defined new branches, new theories. The field of membrane computing was initiated by Gheorghe Păun as a branch of natural computing [3]; P systems are 
inspired by the hierarchical membrane structure of eukaryotic cells [4]. An impressive handbook of membrane computing was published recently (2010) by Oxford University Press.

After 1990 he becomes a traveling scientist, visiting several countries and receiving many research fellowships and awards. Fruitful scientific collaboration at Magdeburg University (Germany), and at University of Turku (Finland). The trio Gheorghe Păun, Grzegorz Rozenberg and Arto Salomaa is well-known for several successful books. The last years were spent in Spain, first in Tarragona and now in Sevilla. Several collaborations were possible during his trips, and there are over 100 co-authors from many countries. His scientific reputation is related to the large number of invited talks provided at many international conferences and universities. He is a member of the editorial boards for several international journals, corresponding member of the Romanian Academy (from 1997), and member of Academia Europaea (from 2006).

It is not possible to understand the personality of Gheorghe Păun without mentioning his activity as writer of novels and poems; he is a member of the Romanian Writers Association for a long time. Another aspect of his life is related to the intellectual seduction of games; he was the promoter of GO in Romania, writing many books about GO and other "mathematical" games.

Personally, I am impressed by the speed of his mind (it is enough to say few words about some new results, and he is able to complete quickly the whole approach), his wide-ranging curiosity and intelligence, rich imagination and humor, talent and passion. He is highly motivated by challenging projects, and work hard to conclude them successfully. There are very few scientists having such an interesting profile, and I am very happy to learn a lot from him.

Celebrating his 60th birthday, we wish him a good health, long life, and new interesting achievements!

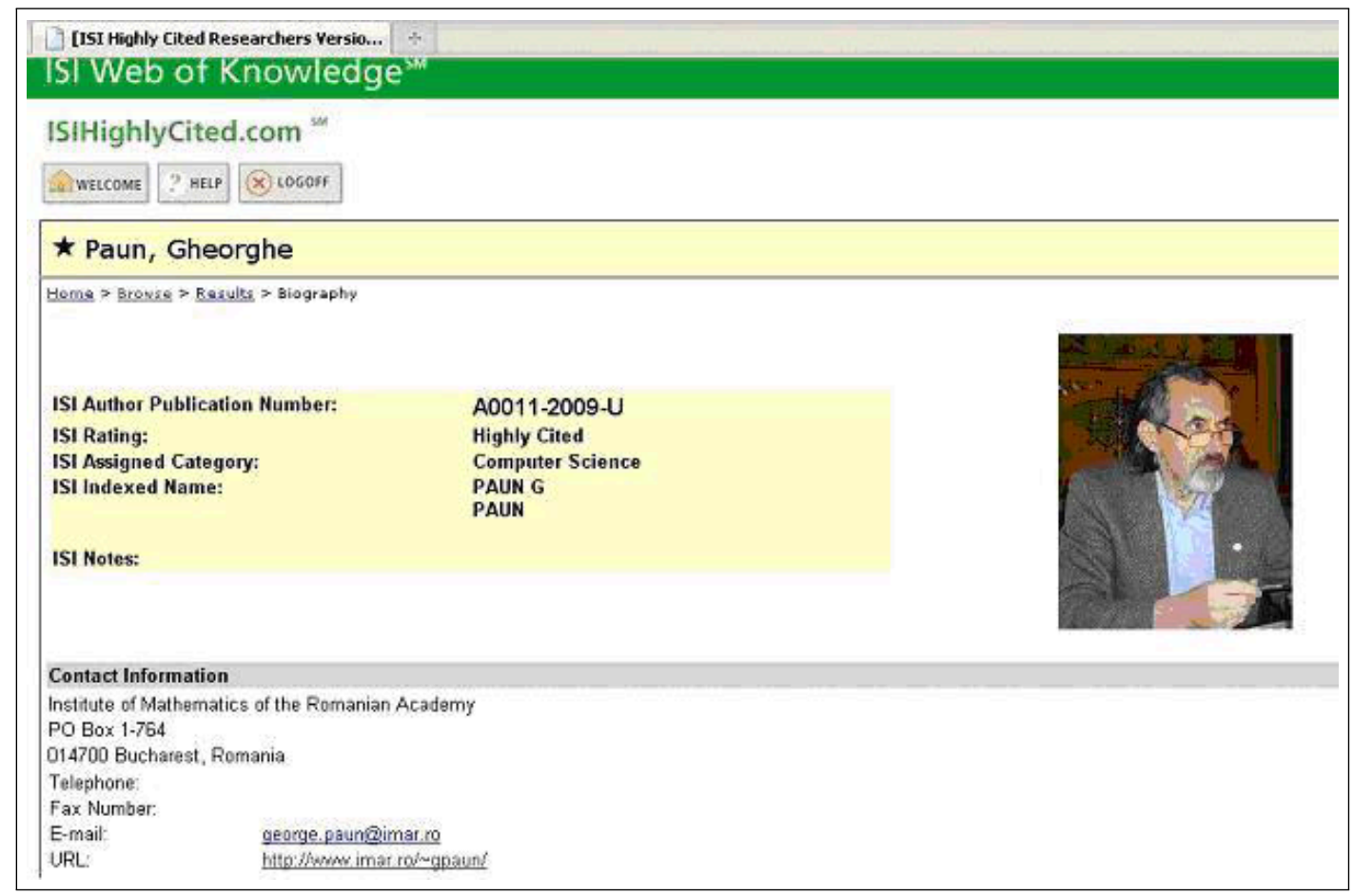

Figure 2: G. Păun-An ISI Highly Cited Researcher (A copy cropped from [5]) 


\section{Bibliography}

[1] Gheorghe Păun, One More Universality Result for P Systems with Objects on Membranes, International Journal of Computers, Communications \& Control, 1(1): 25-32, 2006 (Free access at http://www.journal.univagora.ro/download/pdf/21.pdf)

[2] http://www.imar.ro/ gpaun/

[3] http://esi-topics.com/

[4] http://ppage.psystems.eu/

[5] http://hcr3.isiknowledge.com/

[6] http://interaction.lille.inria.fr/ roussel/projects/scholarindex/index.cgi 